\title{
A Novel Method for EOG Features Extraction from the Forehead
}

\author{
Hao-Yu Cai, Jia-Xin Ma, Li-Chen Shi and Bao-Liang Lu* Senior Member, IEEE
}

\begin{abstract}
We have shown that the slow eye movements extracted from electrooculogram (EOG) signals can be used to estimate human vigilance in our previous work. However, the traditional method for recording EOG signals is to place the electrodes near the eyes of subjects. This placement is inconvenient for users in real-world applications. This paper aims to find a more practical placement for acquiring EOG signals for vigilance estimation. Instead of placing the electrodes near the eyes, we place them on the forehead. We extract EOG features from the forehead EOG signals using both independent component analysis and support vector machines. The performance of our proposed method is evaluated using the correlation coefficients between the forehead EOG signals and the traditional EOG signals. The results show that a correlation of 0.84 can be obtained when the users make 14 different face movements and for merely eye movements it reaches 0.93 .
\end{abstract}

\section{INTRODUCTION}

Many studies of vigilance in the past few decades have shown that alertness monitoring is very useful for many jobs, especially for vehicle drivers and high accuracy operators [1]. For example, fatigue has been shown to be a serious problem for high speed railway operators [2]. In this case, it is critical to develop a practical system for continuously monitoring operators' alertness level, without disturbing their normal activities.

A number of non-contact fatigue detection systems have emerged in recent years. Most of these systems use image processing and computer vision techniques, adopting a CCD video camera fixed in a cab to recognize whether drivers are tired [3], [4]. The drivers' alertness level is evaluated by monitoring their eye states. These systems mainly detect blink frequency and duration to recognize the drivers' drowsy state. Compared to image-based systems, fatigue detection based on EOG signals can utilize more features of eyelid movements like the accurate eye closing time, the strength and speed of each blink and more importantly the eyeball movements, both slow eye movements (SEMs) and rapid eye movements (REMs) [5]. With all of these additional features, alertness level detection based on EOG signals can offer a more robust and precise estimation. Additionally, in real-world application scenarios, users can cheat the

This work was partially supported by the National Natural Science Foundation of China (Grant No. 90820018), the National Basic Research Program of China (Grant No. 2009CB320901), the Science and Technology Commission of Shanghai Municipality (Grant No. 09511502400), and the European Union Seventh Framework Programme (Grant No. 247619).

H.Y.Cai, J.X.Ma and B.L.Lu are with the Center for Brain-Like Computing and Machine Intelligence, Department of Computer Science and Engineering, Shanghai Jiao Tong University and MOE-Microsoft Key Laboratory for Intelligent Computing and Intelligent Systems, Shanghai Jiao Tong University, 800 Dong Chuan Road, Shanghai 200240, China. ${ }^{*}$ Corresponding author: blluesjtu.edu.cn image-based monitor systems by deliberately controlling eye blinks, however, SEMs are extremely difficult to fake. Hu and Zheng [6] have successfully developed an EOG based drowsiness detection model, but it uses only blink features.

In our previous work, we proposed a method using EOG features, mainly slow eye movements (SEMs), to estimate human alertness [7]. Since EOG signals are much stronger than electroencephalogram (EEG) signals, a method based on EOG is robust and easy applied. In addition, detecting an EOG signal requires only a few electrodes on the face, which means lower requirements for the signal gathering in most cases. The performance evaluation of such method by the correlation coefficients between the estimated alertness level and the local error rates of the subjects shows that the correlation can achieve 0.79 by using EOG features off-line.

This paper introduces a novel electrode placement for EOG detection. Traditionally we use four electrodes, plus a ground electrode, to record EOG signals, with two of them at the outside corners of the eyes to detect horizontal movement and the other two at the top and bottom of one eye for vertical movement; these are called horizontal electrooculogram (HEO) and vertical electrooculogram (VEO), respectively [8]. In practical applications, for instance vehicle driving, contact electrodes close to the eyes are significantly inconvenient to users, especially to spectacle wearers. We rearrange the position of electrodes and gather them all on the forehead. With this arrangement, the electrodes will not impact the sight of the users, which makes the whole device more wearable and users more comfortable.

This paper is organized as follows. Section II describes the EOG detection experimental setup. Section III presents the proposed EOG extraction method using ICA and SVM, and verifies the feasibility of the new placement method. Finally, section IV gives some discussion and conclusions.

\section{MATERIALS AND METHODS}

\section{A. Study subjects}

Our experiment for obtaining forehead EOG signals is a monotonous imitation task. The subject sits about two feet away from an LCD screen in a noise-free room. In each session, the screen displays 14 different face movement examples that would evoke various kinds of EOG and electromyographic (EMG) artifacts. The subject is asked to perform the same action synchronously. All the 14 movements are chosen to represent possible human behaviors during vehicle driving; they include bending or shaking the head, clenching the teeth, blinking, eye rolling, horizontal or vertical eye movement, swallowing, squinting, eyebrow 
raising, scowling, smiling, cheek scratching, and opening and closing the mouth.

The stimuli prompting the subjects to perform different movements are presented in random order. The structure of one trial is as follows: three seconds of counting down on a ready screen for preparation, then the instruction generating the specific movement is presented for 20 seconds. Finally, each trial ends with a brief black screen for relaxation. One experiment contains two sessions, each about 12 minutes, separated by a 5 minute rest.

\section{B. Experimental protocol}

Ten sessions of EOG signals and response sequences from five subjects (four male and one female, all healthy and between 21 and 29) were recorded using the NeuroScan System. In order to evaluate the performance of the two electrode placement methods, both were used at the same time. That is, in addition to reference and ground electrodes, four electrodes were used to record HEO and VEO in the traditional way, and four more were used on the forehead. More details will be mentioned in section III. The recordings are sampled at $500 \mathrm{~Hz}$, and filtered between 0.1 and $100 \mathrm{~Hz}$.

\section{Placement methods}

Although placing electrodes near the eyes can obtain the most direct and noise-free EOG signals, this method has many limitations in practical applications. Thus we try to find a new placement method based on the following criteria.

First, electrodes cannot affect normal operations or the sight of subjects. Second, electrodes should be easy to wear, like wearing a cap or band on the head. Third, given the electrode placement, the detection system should have a certain robustness, for example, it should avoid interference from artifacts or deliberate actions of subjects.

Taking account all of these constraints, we decided that it is best to put electrodes on the forehead. Among the facial muscles, the forehead has less motion than most. It is normally bare so electrodes are not disturbed by hair on the scalp. It is also close to the eyes. Moreover, in this way it is really easy to implement all the electrodes on one band which makes the device quite practicable.

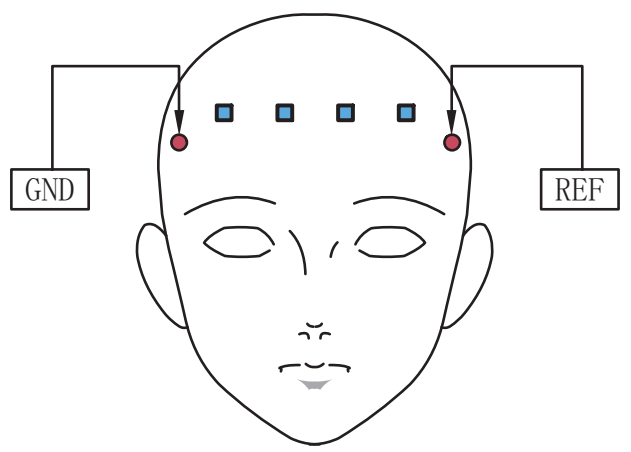

Fig. 1. The far left and far right electrodes on the edge of the forehead are GND and REF. The other four electrodes in the middle of the forehead are observation electrodes.
In our design six electrodes are used to detect EOG signals from forehead. One of them is a reference electrode (Ref), another is ground (Gnd), and the rest are observation electrodes.

Our final placement method is depicted in Fig.1. For easy deployment, the Ref and Gnd were placed on the left and right edges of the forehead respectively and the four observation electrodes were placed equidistant horizontally in the center.

\section{Choice of ICA algorithm}

The first and most important decision that has to be made is which ICA algorithm should be used for the source separation. This is meaningful because an unsuitable source separation algorithm may lead to poor results like fractions of artifacts mixed with the desired EOG signals, as well as confusing HEO and VEO.

We apply two different algorithms: FastICA (an ICA algorithm) from the EEGLAB toolbox [9] and Amuse (a blind source separation, BSS algorithm) from the ICALAB toolbox [10]. The main difference between these two algorithms is that FastICA yields truly statistically independent components, while Amuse just returns uncorrelated signals. Fig. 2 illustrates the comparison between the signals separated by ICA and the original HEO and VEO signals detected by the traditional method.

For both FastICA and Amuse, the input data is a matrix containing data from the four channels of observation signals from the rearranged placement, and the output is four channels of signals after separation. We expected that among the output signal channels, one would represent HEO and another VEO.
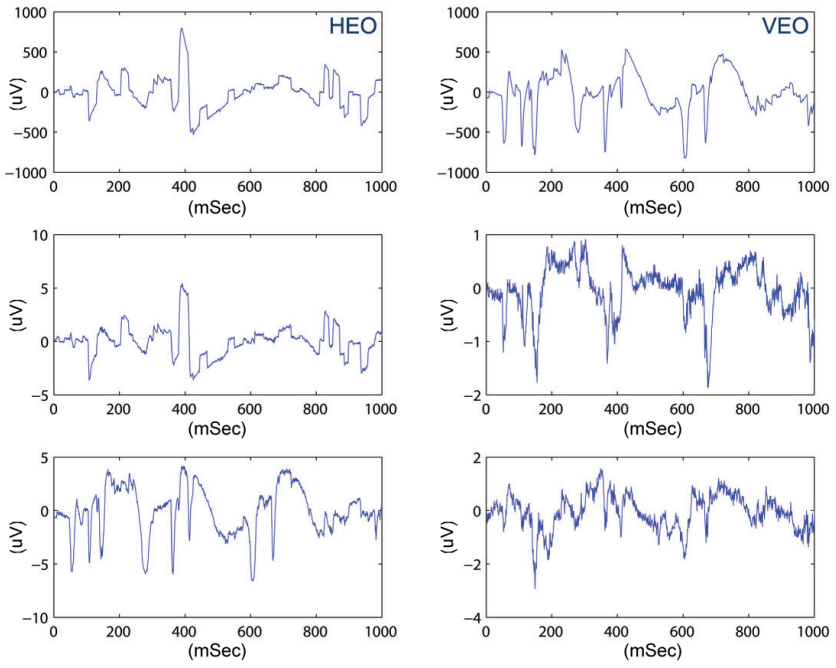

Fig. 2. The signals separated by ICA and the original HEO and VEO signals. The top left image shows HEO, the top right one shows VEO, and the other four are the signals separated by ICA.

In order to evaluate the correlation between the separated signals and the HEO/VEO, FastICA and Amuse are run in two ways. First we use the full length of the whole recordings as the input of both algorithms, and calculate the overall 
correlation coefficients between the four separated signals and HEO or VEO. Second, we divide the recordings into many segments where each corresponds to a 20 second trial (the 3 second rest time in each trial is discarded). Then we run both algorithms independently on each segment and calculate correlation coefficients. In both cases, we select the separated channel which has the largest absolute correlation coefficient with HEO as the representation of HEO, and similarly for VEO. In this case we know both the overall performance of our placement method and the performance for specific movements.

The results show that FastICA can achieve 0.87 overall correlation off-line while Amuse only gets 0.79 . We also notice that for different trials with different movements, the correlation values vary a lot. For trials which only involve eye movements like blinking and eye rolling the correlation value can reach 0.99 , but for other trials, especially those involving forehead movements like scowling, the correlation value is only 0.67 .

Furthermore, after FastICA processing, in most trials the first output channel is most similar to HEO and the third channel to VEO. However, this pattern also changes in some cases. Moreover, comparing the results of the whole session test and the single-trial test, we find that they agree; the channels chosen to represent HEO and VEO are the same in both tests, during trials of strongly eye-related movements. On the other hand, during trials of weakly eye-related movements, the result of a single-trial test may be disturbed and may change to another channel. We will propose a solution to this problem in the next subsection.

The correlation coefficients between signals separated by FastICA and original HEO/VEO for each movement are illustrated in Fig. 3.

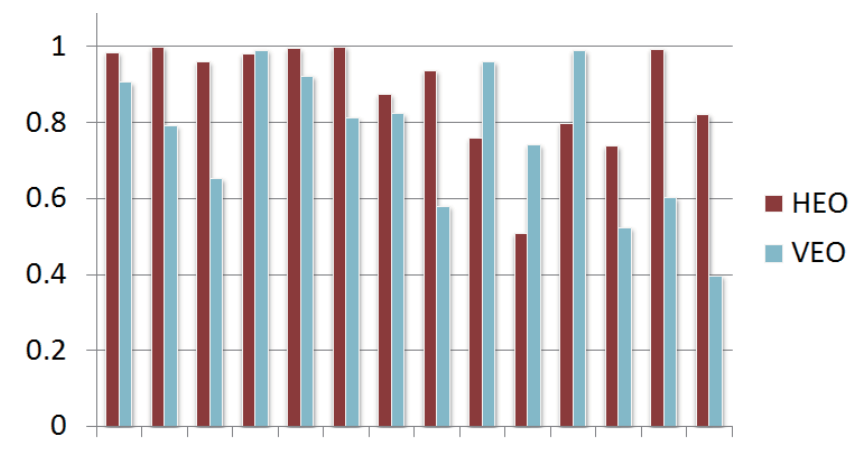

$\begin{array}{llllllllllllll}1 & 2 & 3 & 4 & 5 & 6 & 7 & 8 & 9 & 10 & 11 & 12 & 13 & 14\end{array}$

Fig. 3. The correlation coefficients for each movement.

These 14 different kinds of movements are: 1) bending the head; 2) shaking the head; 3) clenching the teeth; 4) blinking; 5) rolling the eyes; 6) horizontal eye movement; 7) vertical eye movement; 8) swallowing; 9) squinting; 10) raising the eyebrows; 11) scowling; 12) smiling; 13) scratching a cheek; and 14) opening and closing the mouth.

\section{E. EOG Extraction}

As mentioned above, the representation channels for EOG may change for different movements. Even if they are unchanged, in order to correctly extract EOG signal channels from the output of ICA, we still need to find a method which can figure out which channel is most similar to HEO or VEO.

As shown in Fig. 4, we solve this problem in two steps. First, for each output channel, we use a blink detection algorithm to discriminate two possible HEO channels and two possible VEO channels. Second, for all the channels we use a support vector machine (SVM) to generate the probability of belonging to EOG. Then the HEO representation channel should be the one with larger probability in two possible HEO channels, and similarly for VEO.

Normally people have about dozen eye blinks in a minute, and the average interval between two blinks is 4 to 8 seconds. A blink can cause an obvious waveform in VEO, while much less affecting HEO. Therefore, according to the detected number of blinks in signals, we can discriminate possible HEO and VEO. In our experiment there are four channels so we simply select two of them which have larger number of blinks as the VEO candidates, and the other two as the HEO candidates.

Our automatic blink detection method is mainly based on Bruno Jammes's work [11] with some improvements. The core idea of this method is to set two eyelid velocity thresholds: one for eye closing and the other for eye reopening. These two thresholds can be used to locate the positions of blink waveforms in EOG recordings. Since this method works on the differential EOG signal, it can resist the interference of voltage shift caused by muscle movements and so on.

In the next step, we use an SVM to classify all the four channels into two classes which are EOG and EMG. The inputs of the SVM are the features of spectral information and time-series information extracted from each $20 \mathrm{sec}$ segment.

Welch's algorithm is applied to calculate the power spectrum density (PSD) of signals from 0.1 to $100 \mathrm{~Hz}$. The frequency resolution is $5 \mathrm{~Hz}$, which means we divide the signal into 20 bands in the frequency domain and calculate their PSD values. We found the PSD of EOG is predominant at 0.1 to $10 \mathrm{~Hz}$, while the PSD of EMG is mainly distributed above $20 \mathrm{~Hz}$. In this case, EOG and EMG can be distinguished by their typical spectrum features.

Autoregressive (AR) coefficients are used to represent the features of time-series information. EMG differs significantly from EOG in that sudden high-amplitude changes often occur in an EMG signal but seldom appear in an EOG signal. With the help of AR modeling techniques one can detect these sudden changes [12]. Through a 10-fold cross validation $(\mathrm{CV})$ to survey the order $p$ of the AR model from 1 to 8 , we finally choose $p=5$.

Using the features mentioned above as input, an SVM classifier with an RBF kernel [13] is applied on each trial for classifying EOG and EMG. Our SVM classifier was 


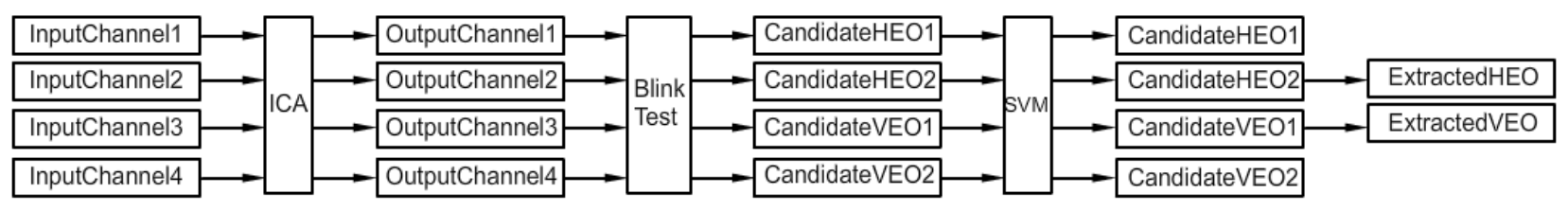

Fig. 4. The process of EOG extraction from the signals detected on the forehead.

trained by 2000 randomly selected samples, half EOG and half EMG. Its classification accuracy is over 90\% [14].

\section{DISCUSSION}

Figure 3 verifies that independent HEO and VEO can be separated from the signals detected at the forehead. In order to estimate the performance of our EOG extraction method made up of SVM and blink detection, we calculated correlation coefficients between the extracted EOG signals and EOG signals detected in the traditional way. When the actual HEO and VEO are already known, the correlation coefficients between ICA separated components and actual HEO/VEO during all the 14 kinds of movements were calculated, and for each trial the channel with the highest correlation coefficient was selected as the representation of HEO or VEO. In this case, we obtained a best sequence of channels representing HEO and VEO, whose correlation value with HEO and VEO is 0.9036 and 0.7862 on average. On the other hand, if we assume the actual HEO and VEO unknown, the representation channels can be selected by our EOG extraction method. The result is about $90 \%$ overlapped with the best sequence. The average correlation values of all the 5 subjects and all the data sessions are 0.8457 for HEO, and 0.7697 for VEO.

The most significant factor causing the decline of correlation values is the EMG interference in some weakly eyerelated movements like teeth clenching and scowling. In such movements, EMG artifacts still exist in the ICA separated signals; this interferes with the SVM classifier and makes it less likely to distinguish EOG from EMG. However, in such a case, even EOG detected in the traditional way may be contaminated by EMG artifacts, which means the correlation value between original EOG and ICA separated EOG may not be a reasonable measure of performance. In normal alertness experiments, subjects do not usually make intense facial muscle movements which continuously generate strong EMG, so we may expect that the actual performance would be higher.

\section{CONCLUSIONS}

In this paper, we have presented a novel electrode placement for EOG detection. With the ICA algorithm the independent HEO and VEO signals can be separated from signals collected from the forehead. Then using SVM combined with a blink detection method, it is possible to select the signals which have the highest correlation with actual HEO or VEO to be the representations of EOG. The final extracted EOG signals can be used to estimate alertness level as mentioned in our previous work. Based on the new electrode placement, alertness monitoring devices can be designed in a more convenient and practical way.

In the future, we will incorporate this electrode placement and EOG extraction method into our real-time alertness estimation system for driving simulation, and continue to enhance the performance of the online EOG extraction algorithm.

\section{REFERENCES}

[1] R. Molloy and R. Parasuraman, "Monitoring an automated system for a single failure: vigilance and task complexity effects", Human Factors, vol.38, pp.311-322, 1996.

[2] J. Dorrian, G. D. Roach, A. Fletcher, and D. Dawson, "Simulated train driving: Fatigue, self-awareness and cognitive disengagement", Applied Ergonomics, vol.38, pp.155-166, 2007.

[3] G. Kaefer, G. Prochart, and R. Weiss, "Wearable Alertness Monitoring for Industrial Applications", Proceedings of the 7th IEEE International Symposium on Wearable Computers, pp.254-255, 2003.

[4] Y. Yang, J. Sheng, and W. Zhou, "Driver's Alertness Level Identify Method Based on Computer Vision", International Conference on Transportation Engineering, vol.246, pp.2386-2391, 2007.

[5] L. D. Gennaro, M. Ferrara, F. Ferlazzo, and M. Bertini, "Slow eye movements and EEG power spectra during wake-sleep transition", Clinical Neurophysiology, vol.111, pp.2107-2115, 2000.

[6] S. Y. Hu and G. T. Zheng, "Driver drowsiness detection with eyelid related parameters by Support Vector Machine", Expert Systems with Applications, vol.36, pp.7651-7658, 2009.

[7] J. X. Ma, L. C. Shi, and B. L. Lu, "Vigilance Estimation by Using Electrooculographic Features", Proceedings of the 32nd International Conference of the IEEE Engineering in Medicine and Biology Society, Buenos Aires, Argentina, Sep., 2010. pp.6591-6594.

[8] R. Barea, L. Boquete, and M. Mazo, "System for assisted mobility using eye movements based on electrooculography", Neural Systems and Rehabilitation Engineering, pp.209-218, 2002.

[9] A. Delorme and S. Makeig, "EEGlab: an open source toolbox for analysis of single-trial EEG dynamics including independent component analysis", Journal of Neuro science Methods, vol.134, pp.9-21, 2004.

[10] A. Cichocki, S. Amari, K. Siwek, T. Tanaka, A. Phan, et al., 'ICALAB toolboxes", http://www.bsp.brain.riken.jp/ICALAB

[11] D. E. B. Jammes and H. Sharabty, "Automatic eog analysis: a first step toward automatic drowsiness scoring during wake sleep transitions", Somnologie, vol.12, pp.227-232, 2008.

[12] T. Ning and J. D. Bronzino, "Autoregressive and bispectral analysis echniques: EEG applications", IEEE Engineering in Medicine and Biology Magazine, vol.9, pp.47-50, 1990.

[13] C. C. Chang and C. J. Lin, "LIBSVM: a library for support vector machines", 2001, http://www.csie.ntu.edu.tw/ cjlin/libsvm

[14] G. Bartels, L. C. Shi, B. L. Lu, "Automatic Artifact Removal from EEG - a Mixed Approach Based on Double Blind Source Separation and Support Vector Machine", Proceedings of the 32nd International Conference of the IEEE Engineering in Medicine and Biology Society, Buenos Aires, Argentina, Sep., 2010. pp.5383-5386. 